\title{
Projection Methods for Dirichlet's Problem in Approximating Polygonal Domains with Boundary-Value Corrections
}

\author{
By James H. Bramble*, Todd Dupont** and Vidar Thomée*
}

\begin{abstract}
Consider Dirichlet's problem in a plane domain $\Omega$ with smooth boundary $\partial \Omega$. For the purpose of its approximate solution, an approximating domain $\Omega_{h}, 0<h \leqq 1$, with polygonal boundary $\partial \Omega_{h}$ is introduced where the segments of $\partial \Omega_{h}$ have length at most $h$. A projection method introduced by Nitsche [6] is then applied on $\Omega_{h}$ to give an approximate solution in a finite-dimensional subspace of functions $S_{h}$, for instance a space of splines defined on a triangulation of $\Omega_{h}$. The boundary terms in the bilinear form associated with Nitsche's method are modified to correct for the perturbation of the boundary.
\end{abstract}

1. Introduction. In this paper, we consider the approximate solution of Dirichlet's problem

$$
-\Delta u=f \quad \text { in } \Omega, \quad u=g \text { on } \partial \Omega,
$$

where $\Omega$ is a bounded plane domain with smooth boundary $\partial \Omega$, and where $f$ and $g$ are given functions in $\Omega$ and on $\partial \Omega$, respectively.

One approach to this problem is to introduce a new domain $\Omega_{h}$ whose boundary $\partial \Omega_{h}$ consists of straight line segments (of maximum length $h$ ) and which is close to $\Omega$. On such an approximate domain, which can be easily subdivided into triangles, some version of the finite element method or Galerkin's method can be applied to define an approximate solution of (1.1). This approach was considered by Nitsche [5] and by Strang and Berger [7] and it was shown that (for a standard Galerkin method) in general the error introduced can be bounded by $C h^{2}$ where $C$ depends on the solution $u$ of (1.1). Thomée [8] has shown that the power of $h$ in their results cannot be improved upon but also that the accuracy can in fact be increased to fourth order by solving a sequence of three problems with successively more refined boundary approximations.

In [6], Nitsche presented a method for solving approximately the original problem (1.1), in which he used a nonstandard bilinear form on the domain $\Omega$. His method does not require that the approximating functions satisfy the boundary condition of (1.1), but his technique demands that the finite-dimensional space of approximating functions satisfy some auxiliary conditions near the boundary. In general, in order to satisfy these conditions (often referred to as inverse conditions) when using piecewise polynomial approximating functions, something special must be done near

Received March 15, 1972.

AMS 1969 subject classifications. Primary 6566.

* Sponsored by the United States Army under Contract No. DA-31-124-ARO-D-462.

** Partially supported by the National Science Foundation.

Copyright @ 1972, American Mathematical Society 
the boundary. However, for domains with polygonal boundaries, these assumptions turn out to be natural. Based on this observation, we present here a method which combines Nitsche's technique with the improved accuracy polygonal domain approximation in [8]. We show that for any natural number $k$ a bilinear form can be constructed such that the corresponding Galerkin method is of order $O\left(h^{k}\right)$ as $h$ tends to zero when applied to appropriate subspaces of approximating functions on the approximating polygonal domain $\Omega_{h}$. These bilinear forms are constructed by modifying the boundary terms in Nitsche's bilinear form to correct for the perturbation of the domain. In the particular cases $k=2$ and 4, the form, and hence the corresponding matrix, can be chosen symmetric.

In Section 2, we introduce notation, state some well-known results on the problem (1.1), and derive some auxiliary estimates needed for the analysis. The main theorems and their proofs are presented in Section 3. In Section 4, we finally discuss the choice of subspaces appropriate for application of the theory.

2. Notation and Preliminaries. Let $\Omega$ be a bounded plane domain with boundary $\partial \Omega$ which we shall assume for convenience to be of class $\mathcal{C}^{\infty}$. We shall suppose that $\Omega$ is approximated by a family of domains $\Omega_{h}, 0<h \leqq 1$, which are contained in $\Omega$ and whose boundaries $\partial \Omega_{h}$ consist of a finite number of line segments. For each fixed $h$, we may write $\partial \Omega_{h}=\bigcup_{i=1}^{M(h)} \partial \Omega_{h}^{(j)}$, where each $\partial \Omega_{h}^{(i)}$ consists of a half open segment and the $\partial \Omega_{h}^{(j)}$ 's are assumed disjoint. With this convention, the outward normal vector $n_{x}$ to $\partial \Omega_{h}$ at $x$ is well defined. We shall suppose that $h=$ $\max _{i}\left\{\right.$ length of $\left.\partial \Omega_{h}^{(j)}\right\}$.

We define, for $x \in \partial \Omega_{h}$,

$$
\delta(x)=\min \left\{s \mid s>0, x+s n_{x} \in \partial \Omega\right\},
$$

and shall always assume that

$$
\delta_{h} \equiv \sup _{x \in \partial \Omega_{h}} \delta(x) \leqq C h .
$$

We make a further technical assumption about the family $\Omega_{h}, 0<h \leqq 1$. For fixed $h$ and $1 \leqq j \leqq M(h)$, define the sets (with the dependence of $h$ suppressed in the notation)

$$
\begin{aligned}
\Omega^{(j)} & =\left\{y \mid y=x+s n_{x}, 0<s<\delta(x), x \in \partial \Omega_{h}^{(j)}\right\}, \\
(\partial \Omega)^{(j)} & =\left\{y \mid y=x+\delta(x) n_{x}, x \in \partial \Omega_{h}^{(j)}\right\} .
\end{aligned}
$$

We assume that there is an integer $N$ independent of $h$ such that each $\Omega^{(j)}\left((\partial \Omega)^{(j)}\right)$ meets at most $N$ of the $\Omega^{(l)}\left((\partial \Omega)^{(l)}\right), l \neq j$. If the domain $\Omega$ is smooth and convex and we take the set $\partial \Omega_{h}$ to have its vertices on $\partial \Omega$, it is obvious that our assumptions are satisfied. In that case, $\delta_{h} \leqq C h^{2}$ and the sets $\Omega^{(j)}$ (and $(\partial \Omega)^{(i)}$ ) are disjoint.

For real-valued functions, we introduce the norms in $L_{2}(\Omega)$ and $L_{2}(\partial \Omega)(x=$ $\left(x_{1}, x_{2}\right), \sigma=$ arc length along $\left.\partial \Omega\right)$,

$$
\|v\|=\left(\int_{\Omega} v^{2} d x\right)^{1 / 2}, \quad|v|=\left(\int_{\partial \Omega} v^{2} d \sigma\right)^{1 / 2},
$$

and denote for $s \geqq 0$ by $H^{s}(\Omega)$ and $H^{s}(\partial \Omega)$ the corresponding Sobolev spaces, defined for $s$ an integer as the closure of $C^{\infty}(\bar{\Omega})$ and $C^{\infty}(\partial \Omega)$ with respect to the norms $\left(D^{\alpha}=\right.$ 
$\left.\left(\partial / \partial x_{1}\right)^{\alpha_{1}}\left(\partial / \partial x_{2}\right)^{\alpha_{2}}, \alpha=\left(\alpha_{1}, \alpha_{2}\right),|\alpha|=\alpha_{1}+\alpha_{2}\right)$

$$
\|v\|_{s}=\left(\sum_{|\alpha| \leqq s}\left\|D^{\alpha} v\right\|^{2}\right)^{1 / 2}, \quad|v|_{s}=\left(\sum_{j \leqq s}\left|\left(\frac{\partial}{\partial \sigma}\right)^{j} v\right|^{2}\right)^{1 / 2},
$$

respectively, and defined for nonintegral $s$ by interpolation (cf. [4]). In Lemma 9, we use the analogous definitions when $\Omega$ is a domain in Euclidean $d$-dimensional space $R^{d}$. We shall also use the corresponding norms, with respect to $\Omega_{h}$ and $\partial \Omega_{h}$,

$$
\|v\|_{s, h}=\left(\sum_{|\alpha| \leqq s} \int_{\Omega_{h}}\left(D^{\alpha} v\right)^{2} d x\right)^{1 / 2}, \quad|v|_{0, h}=\left(\int_{\partial \Omega_{h}} v^{2} d \sigma\right)^{1 / 2} .
$$

In addition to these norms, we shall need the inner products in $L_{2}\left(\Omega_{h}\right)$ and $L_{2}\left(\partial \Omega_{h}\right)$, which we shall denote by

$$
(\varphi, \psi)=\int_{\Omega_{h}} \varphi \psi d x \text { and }\langle\varphi, \psi\rangle=\int_{\partial \Omega_{h}} \varphi \psi d \sigma
$$

respectively, and the Dirichlet form

$$
D(\varphi, \psi)=\int_{\Omega_{h}}\left(\frac{\partial \varphi}{\partial x_{1}} \frac{\partial \psi}{\partial x_{1}}+\frac{\partial \varphi}{\partial x_{2}} \frac{\partial \psi}{\partial x_{2}}\right) d x
$$

We further define, for $k$ a nonnegative integer $(\partial / \partial n$ denotes differentiation with respect to the exterior normal),

$$
\|\varphi\|_{k}=\left(D(\varphi, \varphi)+\sum_{j=0}^{k} h^{2 i-1}\left|\left(\frac{\partial}{\partial n}\right)^{j} \varphi\right|_{0, h}^{2}\right)^{1 / 2},
$$

and let $\mathcal{H}_{k}$ denote the Hilbert space obtained by closure of $C^{\infty}\left(\bar{\Omega}_{h}\right)$ with respect to this norm. It will follow from Lemma 3 below that the restriction to $\Omega_{h}$ of any function in $H^{k+1}(\Omega)$ belongs to $\mathfrak{H}_{k}$.

Notice that although these notions all refer to $\Omega_{h}$, we have suppressed the dependence on $h$ in the notation.

We shall also use the notation

$$
M_{k}(v)=\max _{|\alpha| \leqq k} \sup _{x \in \Omega}\left|D^{\alpha} v(x)\right| .
$$

We state now two well-known results, the proofs of which can be found in e.g. [4].

Lemma 1. For any $s \geqq 0$, there is a constant $C$ such that, for $f \in H^{s}(\Omega), g \in$ $H^{s+3 / 2}(\partial \Omega),(1.1)$ has a unique solution $u \in H^{s+2}(\Omega)$ and

$$
\|u\|_{s+2} \leqq C\left(\|f\|_{s}+|g|_{s+3 / 2}\right) .
$$

We shall always assume below that $f \in L_{2}(\Omega), g \in H^{3 / 2}(\partial \Omega)$, so that $u \in H^{2}(\Omega)$.

LEMMA 2. For any nonnegative integer $m$ and any $\epsilon>0$, there is a constant $C$ such that for $v \in H^{m+1}(\Omega)$ the restriction of $D^{\alpha} v,|\alpha|=m$, to $\partial \Omega$ is well defined and

$$
\sum_{|\alpha|=m}\left|D^{\alpha} v\right| \leqq \epsilon\|v\|_{m+1}+C \epsilon^{-1}\|v\|_{m} .
$$

We shall need the following similar lemma where the estimate is uniform in $h$.

LEMMA 3. For any nonnegative integer $m$ and any $\epsilon>0$, there is a constant $C$ such that $v \in H^{m+1}(\Omega)$ implies $(\partial / \partial n)^{m} v \in L_{2}\left(\partial \Omega_{h}\right)$ for $0<h \leqq 1$ and 


$$
\left|(\partial / \partial n)^{m} v\right|_{0, h} \leqq \epsilon|| v||_{m+1}+C \epsilon^{-1}\|v\|_{m} .
$$

Proof. By choosing local coordinates $(\xi, \eta)$, we may assume for fixed $h$ and $j$ that $\partial \Omega_{h}^{(i)}=\{(\xi, 0) \mid 0 \leqq \xi<\bar{\xi}\}$ and that $\eta>0$ in $\Omega^{(j)}$. We then have $\partial / \partial n=\partial / \partial \eta$ on $\partial \Omega_{h}^{(j)}$. Setting $\delta(\xi)=\delta((\xi, 0))$, we have, for $\varphi=(\partial / \partial n)^{m} v$,

$$
\begin{aligned}
\psi^{2}(\xi, 0) & =\varphi^{2}(\xi, \delta(\xi))-\int_{0}^{\delta(\xi)} \frac{\partial \varphi^{2}}{\partial \eta}(\xi, \eta) d \eta \\
& =\varphi^{2}(\xi, \delta(\xi))-2 \int_{0}^{\delta(\xi)} \varphi(\xi, \eta) \frac{\partial \varphi}{\partial \eta}(\xi, \eta) d \eta,
\end{aligned}
$$

and hence, using obvious estimates and integrating with respect to $\xi$,

$$
\int_{\partial \Omega_{h}^{(i)}} \varphi^{2} d \sigma \leqq \int_{(\partial \Omega(i)} \varphi^{2} d \sigma+\epsilon_{1} \int_{\Omega^{(i)}}\left(\frac{\partial \varphi}{\partial \eta}\right)^{2} d \xi d \eta+\epsilon_{1}^{-1} \int_{\Omega^{(i)}} \varphi^{2} d \xi d \eta,
$$

for any $\epsilon_{1}>0$. The result now follows after summation with respect to $j$, using the assumption about $\Omega^{(i)}$ and $(\partial \Omega)^{(i)}$, and Lemma 2 .

We shall need a similar statement for functions vanishing on $\partial \Omega$. For convex domains and the simplest choice of $\Omega_{h}$, this result is contained in [5].

Lemma 4. There is a constant $C$ such that, for $v \in H^{2}(\Omega)$ with $v=0$ on $\partial \Omega$, we have

$$
|v|_{0, h} \leqq C \delta_{h}|| v \|_{2} .
$$

Proof. We obtain as above

$$
v(\xi, 0)=-\int_{0}^{\delta(\xi)} \frac{\partial v}{\partial \eta}(\xi, \eta) d \eta=-\delta(\xi) \frac{\partial v}{\partial \eta}(\xi, \delta(\xi))+\int_{0}^{\delta(\xi)} \eta \frac{\partial^{2} v}{\partial \eta^{2}}(\xi, \eta) d \eta,
$$

and again after obvious estimates,

$$
\int_{\partial \Omega_{h}^{(i)}} v^{2} d \sigma \leqq 2 \delta_{h}^{2} \int_{(\partial \Omega)(i)}\left(\frac{\partial v}{\partial \eta}\right)^{2} d \sigma+\frac{2}{3} \delta_{h}^{3} \int_{\Omega^{(i)}}\left(\frac{\partial^{2} v}{\partial \eta^{2}}\right)^{2} d \xi d \eta
$$

The result follows as above by summation over $j$.

We shall now introduce the finite-dimensional subspaces in which we shall seek the approximate solution of (1.1). We shall say that the family of finite-dimensional spaces $\left\{S_{h}\right\}_{0<h \leqq 1}$ is of class $J_{k}$ if $S_{h} \subset \mathcal{F}_{k}$ for $0<h \leqq 1$ and if there is a constant $C$ such that

$$
\|\| \varphi\left\|_{k} \leqq C\right\|\|\varphi\|_{0}, \quad \varphi \in S_{h}
$$

This inequality could obviously not be satisfied on the whole of $\mathfrak{H}_{k}$ for $k \geqq 1$ and the restriction to $S_{h}$ is essential. This type of assumption is often referred to as an inverse assumption. We shall return to a discussion of this condition in Section 4.

3. Approximation of Dirichlet's Problem in $\Omega_{h}$. For $k$ a nonnegative integer and $\gamma>0$, we introduce the bilinear form

$$
N_{k, \gamma}(\varphi, \psi)=D(\varphi, \psi)-\left\langle\frac{\partial \varphi}{\partial n}, \psi\right\rangle-\left\langle\sum_{i=0}^{k} \frac{1}{j !} \delta^{j}\left(\frac{\partial}{\partial n}\right)^{j} \varphi, \frac{\partial \psi}{\partial n}-\gamma h^{-1} \psi\right\rangle,
$$

defined for $\varphi \in \mathfrak{H}_{k}, \psi \in \mathfrak{H}_{1}$. Set $\bar{k}=\max (k, 1)$. 
We shall study some properties of $N_{k, \gamma}$. First we have the following:

LeMmA 5. For $\gamma>0$ and $k \geqq 0$ given, there is a constant $C$ such that

$$
\left|N_{k, \gamma}(\varphi, \psi)\right| \leqq C\left|\|\varphi\|_{\bar{k}}\|\psi \psi \mid\|_{1}, \quad \varphi \in \mathfrak{H}_{\bar{k}}, \psi \in \mathfrak{H}_{1}\right.
$$

Proof. Clearly,

$$
\begin{aligned}
\left|D(\varphi, \psi)-\left\langle\frac{\partial \varphi}{\partial n}, \psi\right\rangle\right| & \leqq D(\varphi, \varphi)^{1 / 2} D(\psi, \psi)^{1 / 2}+h^{1 / 2}\left|\frac{\partial \varphi}{\partial n}\right|_{0, h} h^{-1 / 2}|\psi|_{0, h} \\
& \leqq\|\mid \varphi\|_{1}\|\| \psi \|_{0} .
\end{aligned}
$$

Since $\delta_{h} \leqq C h$, we have

$$
\begin{aligned}
\left|\left\langle\delta^{j}\left(\frac{\partial}{\partial n}\right)^{j} \varphi, \frac{\partial \psi}{\partial n}-\gamma h^{-1} \psi\right\rangle\right| & \leqq C h^{i-1 / 2}\left|\frac{\partial^{j} \varphi}{\partial n^{j}}\right|_{0, h}\left(h^{1 / 2}\left|\frac{\partial \psi}{\partial n}\right|_{0, h}+h^{-1 / 2}|\psi|_{0, h}\right) \\
& \leqq C\|\mid \varphi\|\left\|_{j}\right\|\|\psi\|_{1} .
\end{aligned}
$$

Combining (3.1) and (3.2) yields the result.

Considered on $\mathfrak{H}_{k} \times \mathcal{H}_{k}, N_{k, \gamma}$ is not positive definite. However, under certain assumptions on $\Omega_{h}$ we can prove the definiteness of $N_{k, \gamma}$ on $S_{h}$.

Lemma 6. Let $k \geqq 0$ and suppose that $\delta_{h}=o(h)$ as $h \rightarrow 0$ and that $\left\{S_{h}\right\} \in J_{k}$. Then there are positive constants $\gamma, h_{0}$, and c such that, for $0<h \leqq h_{0}$, we have

$$
N_{k, \gamma}(\varphi, \varphi) \geqq c\|\| \varphi \|_{\tilde{k}}^{2}, \quad \varphi \in S_{h} .
$$

Proof. Using the assumption (2.1) (with $k=1$ ), it is easily seen that, for $\gamma$ chosen sufficiently large,

$$
N_{0, \gamma}(\varphi, \varphi) \geqq c\|\| \varphi \|_{1}^{2}, \quad \varphi \in S_{h}
$$

(this was proved by Nitsche in [6]). Using (2.1) again, it follows (with a new $c$ ) that

$$
N_{0, \gamma}(\varphi, \varphi) \geqq c\|\| \varphi \|_{k}^{2}, \quad \varphi \in S_{h} .
$$

Now

$$
N_{k, \gamma}(\varphi, \varphi)=N_{0, \gamma}(\varphi, \varphi)-\left\langle\sum_{j=1}^{k} \frac{1}{j !} \delta^{i}\left(\frac{\partial}{\partial n}\right)^{i} \varphi, \frac{\partial \varphi}{\partial n}-\gamma h^{-1} \varphi\right\rangle .
$$

We obtain from the definition of the norm

$$
\left|\left\langle\sum_{j=1}^{k} \frac{1}{j !} \delta^{i}\left(\frac{\partial}{\partial n}\right)^{j} \varphi, \frac{\partial \varphi}{\partial n}-\gamma h^{-1} \varphi\right\rangle\right| \leqq \tilde{C} \delta_{h} h^{-1}\|\mid \varphi\|_{k}^{2},
$$

where $\tilde{C}$ depends on $\gamma$ but not on $\varphi$ or $h$. Hence, we may conclude that

$$
N_{k, \gamma}(\varphi, \varphi) \geqq\left(c-\tilde{C} \delta_{h} h^{-1}\right)\|\varphi \varphi\|_{k}^{2} .
$$

Since $\delta_{h}=o(h)$ as $h \rightarrow 0$ the result follows.

We now formulate the problem whose solution we will consider as an approximation to the solution $u$ of (1.1): Find $U \in S_{h}$ such that

$$
N_{k, \gamma}(U, \varphi)=(f, \varphi)-\left\langle\tilde{g}, \frac{\partial \varphi}{\partial n}-\gamma h^{-1} \varphi\right\rangle, \text { for all } \varphi \in S_{h},
$$

where $\tilde{g}(x)=g\left(x+\delta(x) n_{x}\right)$. We have 
LEMmA 7. Let $k \geqq 0$ and suppose that $\delta_{h}=o(h)$ as $h \rightarrow 0$ and that $\left\{S_{h}\right\} \in J_{k}$. Further let $\gamma$ and $h_{0}$ be chosen as in Lemma 6. Then, for $0<h \leqq h_{0}$, the problem (3.3) admits a unique solution $U$.

Proof. Uniqueness follows immediately from the positivity of $N_{k, \gamma}$ on $S_{h}$ (Lemma 6). Since (3.3) is a finite-dimensional linear system, existence follows from uniqueness.

We now prove our first estimate for the error $U-u$.

THEOREM 1. Let $k$ be a nonnegative integer and suppose that $\delta_{h}=o(h)$ as $h \rightarrow 0$, $\left\{S_{h}\right\} \in J_{k}$ and that $\gamma$ and $h_{0}$ are chosen as in Lemma 6. Then, there is a constant $C$ such that, if $u \in C^{k+1}(\bar{\Omega})$, we have, for $0<h \leqq h_{0}$,

$$
\|u-U\| \|_{\bar{k}} \leqq C\left\{\inf _{\chi \in S_{h}}\|u-\chi\|_{\bar{k}}+\delta_{h}^{k+1} h^{-1 / 2} M_{k+1}(u)\right\} .
$$

Proof. We consider the case $k \geqq 1$; the case $k=0$ is treated analogously. Let $\chi$ be an arbitrary element of $S_{h}$. Then, by the triangle inequality,

$$
\|u-U\|\left\|_{k} \leqq\right\| u-\chi\|\|_{k}+\|\| \chi-U\|\|_{k} .
$$

Since $\chi-U \in S_{h}$, we obtain, by Lemma 6 ,

$$
\begin{aligned}
\|\chi-U\|_{k}^{2} & \leqq C N_{k, \gamma}(\chi-U, \chi-U) \\
& =C N_{k, \gamma}(\chi-u, \chi-U)+C N_{k, \gamma}(u-U, \chi-U),
\end{aligned}
$$

and because of (3.3),

$$
\begin{aligned}
\left|N_{k, \gamma}(u-U, \chi-U)\right| & \left|\left\langle\tilde{g}-\sum_{j=0}^{k} \frac{1}{j !} \delta^{j}\left(\frac{\partial}{\partial n}\right)^{j} u, \frac{\partial(\chi-U)}{\partial n}-\gamma h^{-1}(\chi-U)\right\rangle\right| \\
& \leqq C \delta_{h}^{k+1} M_{k+1}(u)\left\{\left|\frac{\partial}{\partial n}(\chi-U)\right|_{0, h}+h^{-1}|\chi-U|_{0, h}\right\} \\
& \leqq\left. C \delta_{h}^{k+1} M_{k+1}(u) h^{-1 / 2}|||\chi-U|\right|_{1} .
\end{aligned}
$$

Using Lemma 5, (3.5) and (3.6), we therefore have

$$
\|\| \chi-U\|\|_{k} \leqq C\left(\|u-\chi\| \|_{k}+\delta_{h}^{k+1} h^{-1 / 2} M_{k+1}(u)\right) .
$$

Combining (3.4) and (3.7), we get

$$
\|u-U\| \|_{k} \leqq C\left(\|u-\chi\| \|_{k}+\delta_{h}^{k+1} h^{-1 / 2} M_{k+1}(u)\right) .
$$

Since $\chi$ is arbitrary in $S_{h}$, the result follows.

We want next to study the error $u-U$ in the $L_{2}$-norm. We shall do so by a modification of a method of Nitsche [6]. For this purpose, we need to make a further assumption regarding the spaces $S_{h}$. We thus suppose (cf. Section 4 ) that there is a constant $C$ such that

$$
\inf _{x \in S_{h}}\|v-\chi\|_{1} \leqq C h\|v\|_{2}, \quad v \in H^{2}(\Omega), 0<h \leqq 1 .
$$

We now introduce an auxiliary function $w$ defined as the solution of the Dirichlet problem

$$
\begin{aligned}
-\Delta w=e & \text { in } \Omega_{h}, \\
=0 & \text { in } \Omega \backslash \Omega_{h},
\end{aligned}
$$




$$
w=0 \text { on } \partial \Omega,
$$

where $e=u-U$. We collect some of its properties in the next lemma.

LEMMA 8. There is a constant $C$ such that, for $0<h \leqq 1$,

$$
\begin{aligned}
|w|_{0, h} & \leqq C \delta_{h}|| e \|_{0, h}, \\
|\partial w / \partial n|_{0, h} & \leqq C|| e \|_{0, h},
\end{aligned}
$$

and

$$
\inf _{\chi \in S_{h}}\|w-\chi\|_{1} \leqq C h\|e\|_{0, h} .
$$

Proof. The first inequality (a) is a direct application of Lemmas 1 and 4 to $w$. Part (b) follows from Lemmas 1 and 3, and (c) is a consequence of assumption (3.8) and Lemma 1.

We now have the following:

THEOREM 2. Let $k$ be a nonnegative integer and suppose that $\delta_{h}=o(h)$ as $h \rightarrow 0$, $\left\{S_{h}\right\} \in J_{k}$ and that $\gamma$ and $h_{0}$ are chosen as in Lemma 6 . Then there is a constant $C$ such that, if $u \in C^{k+1}(\bar{\Omega})$, we have for $0<h \leqq h_{0}$,

$$
\|u-U\|_{0, h} \leqq C\left\{\left(h+\delta_{h} h^{-1 / 2}\right)\|\| u-U \|_{\bar{k}}+\delta_{h}^{k+1} M_{k+1}(u)\right\} .
$$

Proof. Using the definition of $w$, Green's identity, and the definition of $N_{k, \gamma}$, we have

$$
\begin{aligned}
\|e\|_{0, h}^{2}= & -(\Delta w, e)=D(w, e)-\langle\partial w / \partial n, e\rangle \\
= & N_{k, \gamma}(e, w)+\left\langle\sum_{j=1}^{k} \frac{1}{j !} \delta^{j}\left(\frac{\partial}{\partial n}\right)^{j} e, \frac{\partial w}{\partial n}\right\rangle \\
& -\gamma h^{-1}\left\langle\sum_{j=0}^{k} \frac{1}{j !} \delta^{j}\left(\frac{\partial}{\partial n}\right)^{i} e, w\right\rangle+\left\langle\frac{\partial e}{\partial n}, w\right\rangle,
\end{aligned}
$$

with the second term on the right omitted when $k=0$. Using Schwarz's inequality and the fact that $\delta_{h} \leqq C h$, we find that

$$
\|e\|_{0, h}^{2} \leqq N_{k, \gamma}(e, w)+C\left\{\delta _ { h } h ^ { - 1 / 2 } \left\|\left|e\left\|_{\bar{k}}\left|\frac{\partial w}{\partial n}\right|_{0, h}+\left.h^{-1 / 2}\left|\|e\|_{\bar{k}}\right| w\right|_{0, h}\right\} .\right.\right.\right.
$$

Hence, using Lemma 8(a), (b), we obtain

$$
\|e\|_{0, h}^{2} \leqq N_{k, \gamma}(e, w)+C \delta_{h} h^{-1 / 2}\|\| e\left\|_{\bar{k}}\right\| e \|_{0, h} .
$$

Now from Lemma 5 and (3.6), we have, for arbitrary $\chi \in S_{h}$,

$$
\begin{aligned}
N_{k, \gamma}(e, w) & =N_{k, \gamma}(e, w-\chi)+N_{k, \gamma}(e, \chi) \\
& \leqq C\left\{\left\||| e||_{\bar{k}}\right\| \mid w-\chi \|_{1}+\delta_{h}^{k+1} M_{k+1}(u)\left(\left|\frac{\partial \chi}{\partial n}\right|_{0, h}+h^{-1}|\chi|_{0, h}\right)\right\} .
\end{aligned}
$$

Adding and subtracting $w$ and using the triangle inequality on the last term, we obtain

$$
\begin{aligned}
N_{k, \gamma}(e, w) \leqq C\{ & \|e\|\left\|_{\bar{k}}\right\| \mid w-\chi \|_{1} \\
& +\delta_{h}^{k+1} M_{k+1}(u)\left(h^{-1 / 2}\left\|\left.\left|w-\chi \|_{1}+\right| \frac{\partial w}{\partial n}\right|_{0, h}+h^{-1}|w|_{0, h}\right)\right\} .
\end{aligned}
$$


Since $\chi$ is arbitrary, we may take the infimum on the right side of (3.10) and then apply Lemma 8(a), (b), (c) to obtain

$$
N_{k, \gamma}(e, w) \leqq C\|e\|_{0, h}\left\{h\|\| e \|_{\bar{k}}+\delta_{h}^{k+1} M_{k+1}(u)\right\} .
$$

Combining (3.9) and (3.11), the result follows.

Except for the case $k=0$, the bilinear form $N_{k, \gamma}$ is nonsymmetric when $\delta \not \equiv 0$. In the case $k=1, N_{1, \gamma}$ may be symmetrized in a simple way with many of its properties unaltered. We define

$$
\begin{aligned}
\tilde{N}_{1, \gamma}(\varphi, \psi)= & D(\varphi, \psi)-\left\langle\varphi, \frac{\partial \psi}{\partial n}\right\rangle-\left\langle\frac{\partial \varphi}{\partial n}, \psi\right\rangle-\left\langle\delta \frac{\partial \varphi}{\partial n}, \frac{\partial \psi}{\partial n}\right\rangle \\
& +\gamma h^{-1}\left\langle\varphi+\delta \frac{\partial \varphi}{\partial n}, \psi+\delta \frac{\partial \psi}{\partial n}\right\rangle .
\end{aligned}
$$

All previous results for $N_{1, \gamma}$ now hold for $\tilde{N}_{1, \gamma}$. The proofs are trivial modifications of those for $N_{1, \gamma}$. The new form has the additional advantageous property that $\tilde{N}_{1, \gamma}(\varphi, \varphi) \geqq \tilde{N}_{1, \gamma_{0}}(\varphi, \varphi)$ for $\gamma \geqq \gamma_{0}$. Hence, if $\tilde{N}_{1, \gamma}$ is positive definite on $S_{h}$ for $0<h \leqq h_{0}$, then so is $\tilde{N}_{1, \gamma}$ for $\gamma \geqq \gamma_{0}$.

We also note that if $N_{k, \gamma}$ is defined using an approximate $\delta(x)$ given by $\sigma(x)$ on $\partial \Omega_{h}$ and $\sigma_{h}=\max _{\partial \Omega_{h}}|\sigma(x)-\delta(x)| \leqq C \delta_{h}$, then Lemmas 5, 6 and 7 hold. Theorems 1 and 2 also hold provided $\delta_{h}^{k+1}$ is replaced by $\left(\delta_{h}^{k+1}+\sigma_{h}\right)$.

4. Application to Particular Subspaces. We shall now introduce some classes of subspaces to which our results conveniently apply.

For given positive integers $k$ and $r$ with $r \geqq k+1$, we say that the family $\left\{S_{h}\right\}_{0<h \leqq 1}$ of finite-dimensional spaces is of class $\delta_{k, r}$, if, for each $h, S_{h} \subset \mathfrak{H}_{k}$ and if there is a constant $C$ such that, for $v \in H^{r}(\Omega), 0<h \leqq 1$,

$$
\inf _{x \in S_{h}}\|v-\chi\|\left\|_{k} \leqq C h^{r-1}\right\| v \|_{r} .
$$

Condition (3.8) clearly means $\left\{S_{h}\right\} \in \delta_{1,2}$. We shall see below that $\delta_{k, r} \subset \delta_{1,2}$.

We first prove an interpolation estimate similar to one which was proved in [2] in a somewhat different setting. In view of possible other applications, we phrase this lemma in a more general form than is needed here.

LEMMA 9. Let $\Omega \subset R^{d}$ be a bounded domain having the restricted cone property. Then, for $0 \leqq k \leqq s \leqq r$ given, there is a constant $C$ such that, for $v \in H^{s}(\Omega)$, $0<h \leqq 1$,

$$
\inf _{w \in H^{r}(\Omega)}\left\{\sum_{j=0}^{k} h^{i}\|v-w\|_{i}+h^{r}\|w\|_{r}\right\} \leqq C h^{s}\|v\|_{s} .
$$

Proof. By the Calderón extension theorem (cf., e.g., [1] for a precise statement and definition of the restricted cone property), there is a constant $C_{0}$ and for $v \in H^{s}(\Omega)$ an extension $v_{e} \in H^{s}\left(R^{d}\right)$ such that

$$
\left\|v_{e}\right\|_{H^{*}\left(R^{d}\right)} \leqq C_{0}\|v\|_{s}
$$

Now let $w_{e} \in H^{r}\left(R^{d}\right)$ be defined by

$$
\begin{aligned}
\hat{w}_{e}(\xi) & =\hat{v}_{e}(\xi), & & h^{2}\left(|\xi|^{2}+1\right) \leqq 1, \\
& =0, & & h^{2}\left(|\xi|^{2}+1\right)>1,
\end{aligned}
$$


where $\hat{v}_{e}$ and $\hat{w}_{e}$ denote the Fourier transforms of $v_{e}$ and $w_{e}$, respectively. We obtain by obvious estimates

$$
\begin{aligned}
& \inf _{w \in H^{r}(\Omega)}\left\{\sum_{j=0}^{k} h^{j}|| v-w \|_{i}+h^{r}|| w||_{r}\right\} \leqq \sum_{j=0}^{k} h^{i}\left\|v_{e}-w_{e}\right\|_{H^{j}\left(R^{d}\right)}+h^{r}|| w_{e} \|_{H^{r}\left(R^{d}\right)} \\
& \leqq C\left\{\int_{R^{d}} \sum_{j=0}^{k} h^{2 j}\left(|\xi|^{2}+1\right)^{i}\left|\hat{v}_{e}-\hat{w}_{e}\right|^{2} d \xi+\int_{R^{d}} h^{2 r}\left(|\xi|^{2}+1\right)^{r}\left|\hat{w}_{e}\right|^{2} d \xi\right\}^{1 / 2} \\
& \leqq C\left\{\int_{h^{2}\left(|\xi|^{2}+1\right)>1}\left[h^{2}\left(|\xi|^{2}+1\right)\right]^{k}\left|\hat{v}_{e}\right|^{2} d \xi+\int_{h^{2}\left(|\xi|^{2}+1\right) \leqq 1}\left[h^{2}\left(|\xi|^{2}+1\right)\right]^{r}\left|\hat{v}_{e}\right|^{2} d \xi\right\}^{1 / 2} \\
& \leqq C\left\{\int_{R^{d}}\left[h^{2}\left(|\xi|^{2}+1\right)\right]^{s}\left|\hat{v}_{e}\right|^{2} d \xi\right\}^{1 / 2} \leqq C h^{s}|| v_{e} \|_{H^{s}\left(R^{d}\right)},
\end{aligned}
$$

and the result therefore follows by (4.1).

We can now prove the following inclusion lemma.

LEMMA 10 . Let $r_{i} \geqq k_{i}+1, j=1,2, r_{1} \leqq r_{2}, k_{1} \leqq k_{2}$. Then

$$
S_{k_{2}, r_{2}} \subset S_{k_{1}, r_{2}} \subset S_{k_{1}, r_{1}} \text {. }
$$

Proof. Assume that $\left\{S_{h}\right\} \in S_{k_{2}, r_{2}}$. We have immediately, for $v \in H^{r}(\Omega)$,

$$
\inf _{\chi \in S_{h}}\|v-\chi\|\left\|_{k_{1}} \leqq \inf _{x \in S_{h}}\right\| v-\chi\|\|_{k_{2}} \leqq C h^{r_{2}-1}\|v\|_{r_{2}}
$$

so that $\left\{S_{h}\right\} \in S_{k_{1}, r_{2}}$. Further, we obtain, using Lemma 3 and the definition of $\delta_{k_{1}, r_{2}}$, for $w \in H^{r_{2}}(\Omega)$,

$$
\begin{aligned}
\inf _{\chi \in S_{h}}\|v-\chi\| \|_{k_{1}} & \leqq \inf _{\chi \in S_{h}}\left\{\|v-w\|\left\|_{k_{1}}+\right\|\|w-\chi\| \|_{k_{1}}\right\} \\
& \leqq C\left\{\sum_{j=0}^{k_{1}+1} h^{i-1}\|v-w\|_{i}+h^{r_{2}-1}\|w\|_{r_{2}}\right\},
\end{aligned}
$$

or since $w$ is arbitrary, using Lemma 9 ,

$$
\begin{aligned}
\inf _{\chi \in S_{h}}\|v-\chi\| \|_{k_{1}} & \leqq C h^{-1} \inf _{w \in H_{r_{2}}(\Omega)}\left\{\sum_{j=0}^{k_{1}+1} h^{j}\|v-w\|_{j}+h^{r_{2}}\|w\|_{r_{2}}\right\} \\
& \leqq C h^{r_{1}-1}\|v\|_{r_{1}} .
\end{aligned}
$$

This shows that $\left\{S_{h}\right\} \in S_{k_{1}, r_{1}}$ and hence concludes the proof of the lemma.

Since the domain considered is assumed to have a smooth boundary, it is always possible to construct $\Omega_{h}$ such that $\delta_{h} \leqq C h^{2}$. Our theorems then yield the following:

THEOREM 3. Let $l$ be a positive integer and assume $\delta_{h} \leqq C h^{2}$. Further, let $f \in H^{2 l}(\Omega), g \in H^{2 l+3 / 2}(\partial \Omega)$ and assume $\left\{S_{h}\right\} \in \mathcal{S}_{l, 2 l+2} \cap \mathfrak{J}_{l}$. Then the error $e=$ $u-U$ satisfies

$$
\|e\|_{0, h}+h\||| e\|_{l} \leqq C h^{2 l+2}\left(\|f\|_{2 l}+|g|_{2 l+3 / 2}\right) .
$$

Proof. This is an immediate consequence of Theorems 1 and 2 with $k=l$, Lemma 1 , and Sobolev's inequality which implies $M_{l+1}(u) \leqq C\|u\|_{2 l+2}$.

We shall now describe some classes of subspaces based on triangulation of $\Omega_{h}$. Suppose that for each $h$ the domain $\Omega_{h}$ is subdivided into triangles $T_{a}, q=1, \cdots, N_{h}$, whose sides have length bounded above by $h$ and below by $\kappa h$ for some positive $\kappa$. Assume further that the angles in the triangulation are bounded below uniformly 
in $h$. Under these assumptions, any triangle in the triangulation can be mapped onto one fixed triangle $T_{0}$ by an affine transformation. The scale of the transformation will be bounded above and below by a fixed multiple of $h^{-1}$, and the Jacobian of the transformation will be similarly bounded above and below by a multiple of $h^{-2}$. As a consequence, it follows from the trace inequality (using standard notation)

$$
\|u\|_{L_{2}\left(\partial T_{0}\right)} \leqq C\|v\|_{H^{1}\left(T_{0}\right)}
$$

that there is another constant $C$ independent of $v, q$, and $h$ such that

$$
\|v\|_{L_{2}\left(\partial T_{q}\right)} \leqq C\left(h^{1 / 2}\|v\|_{H^{1}\left(T_{q}\right)}+h^{-1 / 2}\|v\|_{L_{2}\left(T_{q}\right)}\right) .
$$

Applying this to the boundary terms in $\||\cdot|\|_{m}$, we easily find

$$
\|v\|_{m} \leqq C \sum_{j=0}^{m+1} h^{i-1}\|v\|_{i, h} .
$$

Consider now the family of spaces $V_{h}^{m}$ consisting of splines (by which we shall mean functions which reduce to polynomials on the triangles) of degree $4 m+1$ which were constructed in [3, p. 815]. We shall see that $\left\{V_{h}^{m}\right\} \in S_{m, 4 m+2}$. To see this, let, for $v \in H^{4 m+2}(\Omega), p_{m}(v) \in V_{h}^{m}$ denote its interpolation polynomial described in [3, p. 810]. Then by our assumptions, Theorem 2 in [3] implies that there is a constant $C$ independent of $v, q$, and $h$ such that

$$
\left\|v-p_{m}(v)\right\|_{H^{i}\left(T_{q}\right)} \leqq C h^{4 m+2-i}\|v\|_{H^{4 m+2}\left(T_{q}\right)}, \quad j=0, \cdots, m+1,
$$

and hence after squaring, summing over $q$ and taking square roots, since $p_{m}(v) \in$ $H^{m+1}\left(\Omega_{h}\right)$,

$$
\left\|v-p_{m}(v)\right\|_{i, h} \leqq C h^{4 m+2-i}\|v\|_{4 m+2}, \quad j=0, \cdots, m+1 .
$$

Since, by (4.3),

$$
\inf _{\chi \in V_{h}}\|v-\chi\|\left\|_{m} \leqq C \sum_{j=0}^{m+1} h^{i-1}\right\| v-p_{m}(v) \|_{i, h},
$$

it follows that $\left\{V_{h}^{m}\right\} \in \S_{m, 4 m+2}$.

In the case $m=1, V_{h}^{1}$ consists of quintic splines which are in $H^{2}\left(\Omega_{h}\right)$. In the interior of the domain, the functions in $\mathfrak{H}_{k}$ only need to be once differentiable in $L_{2}\left(\Omega_{h}\right)$ and the functions in $V_{h}^{1}$ then have excessive regularity. We shall now give an example of a family of spaces in $S_{1,4}$ consisting of cubic splines which are only once differentiable in $L_{2}\left(\Omega_{h}\right)$. For this purpose, consider the spaces $Q_{h}$ of cubic splines having for parameters the values of the function and its gradient at the vertices of the $T_{q}$ and the value of the function at the center of gravity of $T_{q}$ (cf. [9] and [3, p. 818]). The functions in $Q_{h}$ are continuous but not generally continuously differentiable across the edges of the triangles. However, by definition, their derivatives are continuous on $\partial \Omega_{h}$, which implies that they belong to $\mathfrak{F}_{1}$. It follows again by (4.3) and trianglewise use of the estimate in $[3$, p. 818$]$ that if $\tilde{p}(v) \in Q_{h}$ is the interpolation polynomial, determined by $v$, then

$$
\inf _{\chi \in Q_{h}}\|v-\chi\|\left\|_{1} \leqq C \sum_{j=0}^{2} h^{i-1}\right\| v-\tilde{p}(v)\left\|_{i, h} \leqq C h^{3}\right\| v \|_{4},
$$

so that $\left\{Q_{h}\right\} \in S_{1,4}$. 
Let us turn to the inverse assumptions. We shall prove first that $\left\{V_{h}^{m}\right\} \in J_{m}$. Obviously, the inequality (2.1) follows if we can prove that, for $\chi \in V_{h}^{m}, 1 \leqq j \leqq m$,

$$
h^{i-1 / 2}\left\|\partial^{j} \chi / \partial n^{i}\right\|_{L_{2}\left(\partial T_{q}\right)} \leqq C\|\operatorname{grad} \chi\|_{L_{2}\left(T_{q}\right)} .
$$

As in the proof of (4.2) above, this follows from the fact that, for polynomials of degree $4 m+1$,

$$
\left\|\partial^{i} \chi / \partial n^{i}\right\|_{L_{2}\left(\partial T_{0}\right)} \leqq C\|\operatorname{grad} \chi\|_{L_{2}\left(T_{0}\right)},
$$

which is obvious, once we note that the expression on the right is a norm in the finite-dimensional space of polynomials of a given degree, modulo constants. Similarly, we can see that $\left\{Q_{h}\right\} \in J_{1}$.

We conclude by remarking that in practical applications of these results, it is likely that the symmetric form $\tilde{N}_{1, \gamma}$, used in conjunction with a space belonging to a family in $\delta_{1,4}$ (e.g. the family $\left\{Q_{h}\right\}$ of cubic splines described above), would be most advantageous. The estimate of Theorem 3 is valid in this case with $l=1$ and hence the resulting scheme has fourth order accuracy.

Mathematics Department
Cornell University
Ithaca, New York 14850

Mathematics Research Center

The University of Wisconsin

Madison, Wisconsin 53706

Department of Mathematics

Chalmers Institute of Technology and the University of Göteborg

Göteborg, Sweden

1. S. Agmon, Lectures on Elliptic Boundary Value Problems, Van Nostrand Math. Studies, no. 2, Van Nostrand, Princeton, N. J., 1965. MR 31 \#2504.

2. J. H. Bramble \& V. ThOMÉE, "Semidiscrete Galerkin methods for parabolic problems," Math. Comp., v. 26, 1972, pp. 633-648.

3. J. H. BRamble \& M. Zlámal, "Triangular elements in the finite element method," Math. Comp., v. 24, 1970, pp. 809-820.

4. J. L. Lions \& E. Magenes, Problèmes aux limites non homogènes et applications.

Vol. 1, Travaux et Recherches Mathématiques, no. 17, Dunod, Paris, 1968. MR 40 \#512.

5. J. Nitsche, "Lineare Spline-Funktionen und die Methoden von Ritz für elliptische Randwertprobleme," Arch. Rational Mech. Anal., v. 36, 1970, pp. 348-355.

6. J. Nitsche, "Ưber ein Variationsprinzip zur Lösung von Dirichlet-Problemen bei Verwendung von Teilräumen, die keinen Randbedingungen unterworfen sind," Abh. Math. Sem. Univ. Hamburg, v. 36, 1971, pp. 9-15.

7. G. Strang \& A. E. Berger, "The change in solution due to change in domain," (To appear.)

8. V. Тноме́E, "Polygonal domain approximation in Dirichlet's problem," J. Inst. Math. Appl. (To appear.)

9. M. Zlámal, "On the finite element method," Numer. Math., v. 12, 1968, pp. 394-409. MR 39 \# 5074. 